Société d'histoire de la révolution de 1848 et des

révolutions du XIXe siècle

$23 \mid 2001$

Nouvelles approches en histoire économique

Jean-Louis ESCUDIER, Edmond Bartissol, 1841-1916. Du canal de Suez à la bouteille d'apéritif

Paris, Éditions du Centre national de la recherche scientifique, 2000, $309 \mathrm{p}$.

Gérard Bonet

\title{
CpenEdition
}

Édition électronique

URL : http://journals.openedition.org/rh19/325

DOI : 10.4000/rh19.325

ISSN : $1777-5329$

Éditeur

La Société de 1848

Édition imprimée

Date de publication : 1 décembre 2001

Pagination : 239-243

ISSN : 1265-1354

Référence électronique

Gérard Bonet, « Jean-Louis ESCUDIER, Edmond Bartissol, 1841-1916. Du canal de Suez à la bouteille

d'apéritif », Revue d'histoire du XIXe siècle [En ligne], 23 | 2001, mis en ligne le 15 octobre 2002, consulté le 22 septembre 2020. URL : http://journals.openedition.org/rh19/325 ; DOI : https://doi.org/10.4000/ rh19.325

Ce document a été généré automatiquement le 22 septembre 2020.

Tous droits réservés 


\section{Jean-Louis ESCUDIER, Edmond Bartissol, 1841-1916. Du canal de Suez à la bouteille d'apéritif}

Paris, Éditions du Centre national de la recherche scientifique, 2000, $309 \mathrm{p}$.

\section{Gérard Bonet}

Edmond Bartissol est un personnage hors normes. Né en 1841 dans une famille de maçons, à Portel, dans l'Aude, autodidacte, fort d'une solide expérience et de précieuses relations acquises comme conducteur de travaux sur le chantier du canal de Suez, il devient, à partir de 1880, l'un des principaux entrepreneurs de travaux publics du XIX'e siècle au même titre qu'un Gustave Eiffel. Cet aspect du personnage, le plus important puisqu'il lui permit d'asseoir sa toute puissance financière, est aujourd'hui bien oublié en Roussillon, sa terre d'élection dans tous les sens du terme. La mémoire collective perpignanaise ne se rappelle que du Bartissol, affairiste qui œuvra en 1904-1905 au dérasement d'une partie des remparts de la ville, moyennant de fructueux bénéfices. Pourtant, l'homme et le capitaine d'industrie valent mieux que cette caricature.

Employé en 1858 sur le chantier de la ligne de chemin de fer de Narbonne à Perpignan dont son frère Jean a obtenu une partie du marché de la construction, Edmond Bartissol intègre en 1860 le service départemental des Ponts et Chaussées des Pyrénées-

Orientales. En 1863, il quitte l'administration pour se faire embaucher par la Compagnie des chemins de fer du Midi des frères Pereire, Le grand tournant de sa vie, il le connaît en 1866 lorsqu'il s'embarque pour Suez. Là l'attend un poste de conducteur de travaux sur le gigantesque chantier du canal conçu par Ferdinand de Lesseps. En 1870, lorsqu'il rentre en France, il a acquis une formation technique de premier ordre, côtoyé les plus grands entrepreneurs de travaux publics du moment et appris l'importance des relations personnelles. Après des missions d'étude sur l'organisation des chemins de fer en Galicie, dans l'Empire Austro-Hongrois, puis en Turquie et une implication dans l'industrie minière au Portugal, en 1879 l'homme est fin prêt pour s'impliquer dans un 
projet d'une toute autre importance : la construction de la ligne de chemin de fer de la Beira Alta qui traverse le Portugal d'est en ouest, de Figueira da Foz à Vilar Formoso, Ce pays sera l'Eldorado de Bartissol et la terre de ses exploits techniques. Le premier consiste dans la réalisation du port de Lexoès, près de Porto, qui verra en 1886 l'utilisation d'une grue géante mobile Titan de la Compagnie Fives-Lille pour l'exécution des travaux de la jetée. Cet engin "était conçu pour soulever des blocs de 50 tonnes et les transporter à une distance de 29 mètres du point de rotation" précise Jean-Louis Escudier, Entre 1886 et 1890, toujours avec son complice Alexis Duparchy rencontré à Suez, il réalise la ligne de chemin de fer de Belem à Cascais et le grand tunnel du Rossio dans le cadre de l'établissement du chemin de fer métropolitain de Lisbonne. À l'affût de desseins toujours plus ambitieux, il soumet aux autorités du pays un projet colossal de pont métallique sur le Tage. Destiné au trafic routier et ferroviaire, cet ouvrage d'art long de 2 kilomètres devait autoriser "le passage des plus hauts navires grâce à des arches de 300 mètres de largeur et de 48 mètres de hauteur". Une telle réalisation --qui devait contribuer au décollage économique des régions méridionales du Portugal-- d'un coût d'environ 50 millions de francs dépasse les possibilités du pays. "De fait", rappelle Escudier, "Lisbonne ne sera équipée d'un pont sur le Tage qu'en 1965 !" L'essentiel de ses intérêts et de ses relations étant au Portugal, Bartissol se lance à la fin des années 1880 dans l'aventure coloniale. "Au Mozambique, Bartissol s'évertua quinze ans durant à aménager, à obtenir des concessions minières, agricoles ou portuaires et cette orientation lui valut bien des déboires". Au cours de ces mêmes années 1880, il acquiert le domaine royal de Pinheiro, à l'embouchure du Sado, et crée un vignoble de 500 à 600 hectares dont il commercialise la récolte, de l'ordre de 12000 hectolitres, sous l'appellation Bartissol Royal-Pinheiro. Apprécié par le régime portugais, outre plusieurs distinctions, Bartissol est honoré en 1889 d'un titre nobiliaire : celui de vicomte.

En 1892, lorsque le scandale politico-financier du canal de Panama éclate, l'essentiel des intérêts d'Edmond Bartissol est au Portugal "et ses horizons lointains sont plutôt ceux de l'Afrique australe que ceux de l'Amérique centrale" souligne Jean-Louis Esculier. Mais il ne fait aucun doute qu'il a suivi, sinon dès 1879 du moins depuis 1886 par l'intermédiaire de son ami Dauderni parti réaliser une partie du chantier, l'évolution puis les errements de l'entreprise de Ferdinand de Lesseps à Panama. En 1893, ambitionnant "de prendre la tête d'une des affaires industrielles et financières les plus importantes du siècle", il se jette dans un premier projet de relance du chantier qui vise à relier par une ligne de chemin de fer les deux tronçons du canal réalisés sur les deux versants atlantique et pacifique. L'affaire échoue sans surprise pour un Bartissol qui "faisait sien le principe selon lequel il est toujours bon de poser suffisamment haut ses prétentions initiales pour garder une marge de négociation". Un an plus tard, s'engageant à présenter un projet répondant à la volonté d'achèvement du canal, il fonde une compagnie, dont le capital est dans un premier temps de 60 millions. Il engage sa fortune personnelle dans l'affaire pour cinq millions de francs-or soit plus de 100 millions de francs actuels. "On mesure ici tout à la fois la dimension des profits réalisés par Bartissol lors de ses précédentes opérations financières, industrielles et commerciales au Portugal et le challenge qu'il est prêt â relever. Si l'affaire échoue, il sera ruiné". Las, la commission technique chargée parle liquidateur Gaudron d'examiner le projet le rejette sans appel. "En tentant de jouer les premiers rôles dans ce curieux ballet que fut l'affaire de Panama, Bartissol contrariait les intérêts de nombreux hommes d'affaires et d'industriels" écrit Jean-Louis Escudier. L'infortune 
panaméenne de 1894 ne décourage pas Bartissol, tout au contraire. Le voici à Salonique en 1896 où il obtient la construction et la concession du port.

La stratégie internationale d'Edmond Bartissol n'exclue pas la recherche d'un enracinement local. Précurseur, dès 1882 il avance l'idée d'un projet de barrage sur la Têt, à Vinça, afin d'assurer l'irrigation de la plaine du Roussillon. Il envisage la même chose pour les vallées voisines de l'Agly et du Tech, Le projet global d'irrigation de Bartissol n'aboutira pas. Il faudra attendre 1978 et 1994 pour voir l'édification d'une barrage sur la Têt puis sur l'Agly. La première opération immobilière et industrielle de Bartissol voit le jour au centre de Perpignan, tout à côté de la cathédrale Saint-Jean, en 1887. Sur la situation de l'ancien hôpital de la Miséricorde, il construit un ensemble résidentiel de luxe dénommé Cité Bartissol, toujours prisé de nos jours, Dans son enceinte il fonde, en 1893, la fabrique de papier à cigarette Suez. Largement concurrencée par les sociétés Job et Nil des Bardou, Bartissol vendra l'entreprise et la marque dix ans plus tard. À la fin des années 1880 , dans le même périmètre, il construit sur l'emplacement de l'église de Saint-Jean-le-Vieux une usine électrique. En 1898 elle permettra à Perpignan de s'éclairer à l'électricité et d'alimenter, deux ans plus tard, le réseau de tramways qui dessert la ville. L'année 1901 voit le déclassement des fortifications qui enserrent le chef-lieu du Roussillon. La destruction des remparts et l'aménagement urbain de l'espace ainsi libéré sont à l'ordre du jour. Bartissol est le seul entrepreneur de travaux publics local capable de supporter financièrement une opération de cette envergure. L'homme d'affaires s'engage à verser sur cinq ans les 1,3 millions de francs demandés par l'État en payement des 42 hectares libérés. En contrepartie, pour le prix de la démolition, il récupère au cœur de la ville, en zone constructible, 17 des 42 hectares --une opération extrêmement fructueuse. Les 25 hectares restant reviennent, sans bourse délier, à la municipalité. Quant au dérasement des remparts, il provoque une polémique qui, aujourd'hui encore, alimente certaines conversations...

Edmond Barrissol fait son entrée dans l'arène politique à l'occasion des élections législatives de septembre 1889. Radical par accident dans le sillage de son avocat Auguste Cayrol, c'est de justesse qu'il est élu député dans la circonscription de Céret au second tour de scrutin devant le républicain progressiste Jules Pams. "En quatre années de mandat, le bilan d'activité du député Bartissol est relativement modeste. Surtout aucun des dossiers sur lesquels il s'est penché ne concerne directement le Roussillon, donc ses électeurs". Aussi, désormais républicain modéré, est-il battu en août 1893 par son adversaire Pams, encore progressiste. En effet, contrairement à l'affirmation de Jean-Louis Escudier, le futur candidat à la présidence de la République en 1913 ne ralliera le radicalisme qu'avec les législatives de mai 1898. Un scrutin qui voit Bartissol se présenter à Narbonne, sa circonscription d'origine. Élu avec 49 voix d'avance sur son adversaire, le socialiste Ferroul, il sera invalidé fin 1898. Quatre ans plus tard, soutenu par L'indépendant des Pyrénées-Orientales, l'influent quotidien républicain modéré local hostile au Bloc des gauches, il sera confortablement élu dans l'arrondissement de Perpignan. Réélu en 1906, candidat en 1910, "au soir du premier tour de scrutin, nettement devancé il se retire sans gloire" relève Escudier.

Député dans ces années où la viticulture "envahit" le Parlement --en février 1905 il dépose en vain "une proposition de loi tendant à instaurer une déclaration de récolte afin de lutter contre la fraude par sucrage et mouillage des vins à la propriété"-Bartissol trouve, à plus de soixante ans, l'occasion d'un "rebond industriel" dans la vente du vin. Propriétaire de plusieurs domaines dans les Pyrénées-Orientales et 
l'Aude, dont celui de Sériège, il crée en 1904 la Société des vins de Banyuls naturels (SVBN) qui fédère dans un premier temps 442 propriétaires adhérents. "Le second étage du dispositif est une société purement commerciale qui, pendant une durée de trente ans, s'engage à acheter le vin aux adhérents à un prix fixé, assure la mise en bouteilles et commercialise". Sur ce modèle, il s'efforce de mettre sur pied le trust des vins du Midi pour lutter contre la détérioration des cours. Bien reçu dans les PyrénéesOrientales, le "trust Bartissol" ne fait pas l'unanimité dans l'Aude et l'Hérault. Tandis que ce projet est abandonné, la SVBN prospère et personnalise le vin doux naturel qu'elle commercialise en Banyuls Bartissol. 85 ans après la mort d'Edmond Bartissol le Bartissol, après bien des tribulations, désormais propriété du groupe Cusenier reste, en dépit d'un tassement des ventes, la cinquième marque française d'apéritif.

Dans un ouvrage captivant au style fluide, mêlant biographie et monographie, JeanLouis Escudier étudie avec brio le destin exceptionnel d'un bâtisseur aujourd'hui méconnu. Sorti du rang, le "Rothschild des travaux publics" comme le qualifièrent certains de ses contemporains, fut différent en tout. Méfiant, intuitif et pragmatique comme le sont tous les autodidactes --Escudier récuse formellement la formule de self made man-- Edmond Bartissol demeura sa vie durant un franc-tireur dans une société dont il avait forcé la porte, dans une profession dont il ne respectait pas les règles du jeu. Son système financier "juridiquement décentralisé et technique concentré", basé sur la polyvalence des capitaux et des hommes mais encore sur une confiance réciproque et la compétence, finalement de type clanique, était certes souple mais risqué. En un mot aventureux. Rien d'étonnant pour un homme qui, sans être parti de rien --Escudier insiste également sur ce point dans sa conclusion-- n'avait pas grand chose au départ ; pour un homme détaché des contingences matérielles même si en 1883, à 47 ans, il s'offrit le château de Fleury-Mérogis, une petite commune de Seine-etOise dont il fut le "seigneur" et le maire de 1892 jusqu'à sa mort en 1916. Fier de ce domaine de 451 hectares, suffisamment ostentatoire pour que nul ne doute de sa puissance, où il recevait Clémenceau et Joffre, Bartissol l'hypothéqua lorsque nécessaire. Finalement, le grand échec de sa vie fut de n'avoir pas su fonder une dynastie. Sans descendance directe, la nature même de son patrimoine industriel ne se prêtait guère, comme le souligne Jean-Louis Escudier, à la continuation de son activité. Personnage véritablement singulier, Edmond Bartissol méritait cette réévaluation fructueuse, claire et intelligente. 